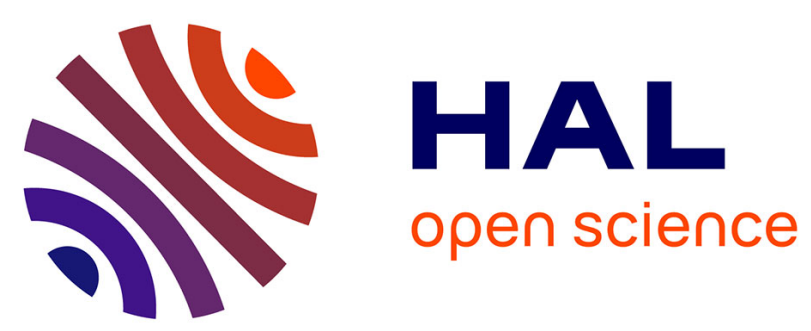

\title{
Mémoires, lieux et territoires
}

Anne Sgard

\section{To cite this version:}

Anne Sgard. Mémoires, lieux et territoires. Mémoires, lieux et territoires, Oct 2004, Rennes, France. pp.105-117. halshs-00325130

\section{HAL Id: halshs-00325130 \\ https://shs.hal.science/halshs-00325130}

Submitted on 26 Sep 2008

HAL is a multi-disciplinary open access archive for the deposit and dissemination of scientific research documents, whether they are published or not. The documents may come from teaching and research institutions in France or abroad, or from public or private research centers.
L'archive ouverte pluridisciplinaire HAL, est destinée au dépôt et à la diffusion de documents scientifiques de niveau recherche, publiés ou non, émanant des établissements d'enseignement et de recherche français ou étrangers, des laboratoires publics ou privés. 
Anne SGARD

Géographe

Laboratoire PACTE-Territoires, Grenoble.

\section{Mémoires, lieux et territoires}

Si la mémoire a été longtemps une problématique centrale de l'histoire, épaulée par la philosophie, elle intéresse aujourd'hui l'ensemble des sciences sociales et le traditionnel partage des tâches avec l'histoire : à celle-ci le temps, son histoire, sa mémoire, à celle-là l'espace et son organisation, a vécu ${ }^{1}$. Les historiens se penchent sur les territoires et les lieux, les géographes s'interrogent sur les temporalités des sociétés et de leurs territoires. Il n'y a pas pour autant de confusion, chaque discipline en se saisissant d'un objet le transforme, le reformule pour l'ajuster à son regard et à ses questionnements. La géographie s'est depuis longtemps saisie de la problématique des temporalités et l'on pense en particulier aux apports de J.-L. Piveteau; de manière plus diffuse, à travers les réflexions sur l'identité avant tout, mais aussi sur le patrimoine, sur le paysage, sur l'appartenance, la question de la dimension temporelle des territoires et de l'usage du passé est bien présente. Si l'on resserre le champ sur la mémoire, bien des pistes restent à explorer, sur lesquelles le géographe peut apporter un éclairage original à travers deux concepts centraux de la géographie: territoire et identité.

Le regard du géographe, renverse et déplace la perspective de l'historien, puisqu'il part du discours des acteurs d'aujourd'hui sur les territoires, sur leur devenir, sur les recompositions en cours, pour y rechercher les logiques d'action. C'est donc moins le contenu de cette mémoire collective ${ }^{2}$ qui l'intéresse, que les situations et les processus actuels de mobilisation de la mémoire par les acteurs. C'est pourquoi, plutôt que de mémoire collective, c'est de construction mémorielle dont il sera question ici. La finalité dès lors n'est pas de la confronter à une histoire scientifique, objectivante, mais de la saisir, comme « un phénomène toujours actuel »(P. Nora), comme un élément de compréhension de la construction des territorialités. L'enjeu n'est donc ni historique, ni patrimonial : il ne s'agit pas d'identifier une mémoire collective à préserver et transmettre ; l'enjeu est identitaire : en partant du postulat que l'identité collective se construit sur des territoires, si ce n'est matériellement maîtrisés en tout cas symboliquement appropriés, la question est de comprendre en quoi la construction mémorielle en tant que processus collectif contribue à l'identification des habitants à un territoire.

Dessinons d'emblée un premier questionnement, en jouant sur les deux mots de territoire et de lieu, ce dernier immédiatement depuis $\mathrm{P}$. Nora ${ }^{3}$ rattaché au thème de la mémoire, mais en usant différemment de ce vocable.

\footnotetext{
${ }^{1}$ Il est néanmoins significatif que dans le Dictionnaire proposé par J.Lévy et M.Lussault (2003), 1'article "mémoire " figure mais soit rédigé par un historien, Patrick Garcia; celui-ci évoque exclusivement les approches historiennes et invite en fin d'article les autres sciences sociales à apporter leur contribution.

${ }^{2}$ Je n'envisagerai ici que la mémoire dans ses dimensions collectives, c'est-à-dire au-delà du cercle familial, non pour faire l'impasse sur des mémoires considérées comme secondaires, mais parce que l'étude des interactions entre ces sphères nous conduirait à dépasser largement les limites de ce présent exercice.

3 Je rappellerai simplement la formule de P. Nora : « enfermer un maximum de sens dans le minimum de signes » (Nora, 1984-1993).
} 
L'observation des pratiques commémoratives laisse penser que la mémoire a besoin de s'inscrire dans des lieux, dans des territoires pour perdurer, dans l'espoir que cette inscription suffise à conjurer l'oubli. Par un renversement un peu facile, on pourrait dire que la géographie se penche alors sur la mémoire des lieux plus que sur les lieux de mémoire.

Parallèlement, les habitants appuient -en partie- la construction de leur identité territoriale sur cette mémoire des lieux, sur la lecture quotidienne du paysage, sur la recherche dans le territoire des signes d'un passé commun, fédérateur. De quelle nature sont les liens entre le projet de territoire, abordé comme la projection du territoire dans le futur, et cet ancrage dans le passé? Les divers acteurs qui investissent un même territoire puisent dans ce fonds et construisent des lectures elles aussi diverses du passé, qui se confrontent et parfois se concurrencent, et cherchent en même temps à se mettre en scène, à se donner à lire. L'inscription dans les lieux de cette mémoire qui valorise des éléments pour en effacer d'autres, qui sélectionne, trie, codifie et parfois invente, devient un enjeu essentiel.

Entre profusion de commémorations, de patrimonialisation, de recours au passé et injonction au projet, à la durabilité voire à la prospective, se construit une articulation entre relecture du passé, diagnostic du présent et anticipation. ${ }^{4} \mathrm{C}^{\prime}$ est bien dans cette problématique qu'est travaillée la question de la mémoire pour le géographe, qui tente de démêler les interactions constantes et étroites entre lecture et écriture des territoires.

\section{Temporalité des territoires : comment construire une continuité entre mémoire et anticipation?}

Les philosophes partagent assez largement une conception des sociétés actuelles comme des sociétés du présent, voire de" l'hyperprésent", vivant dans l'urgence et dans la consommation de l'immédiat (J. Chesnaux, F. Hartog, Z. Laïdi,). Chacun s'accorde aussi généralement à constater la consommation frénétique de passé, par le biais du patrimoine et des commémorations, ou par les diverses manifestations du fameux «devoir de mémoire », ou plus matériellement par la revalorisation des techniques traditionnelles et autres produits de terroirs. P. Garcia a relevé les enjeux bien autres que la seule référence à un événement passé, qui se jouent dans l'acte de commémoration: commémorer " ne met pas seulement en jeu les représentations du passé mais aussi celles de l'avenir et du présent. (...). C'est produire un discours, mettre en scène un geste qui utilise le passé pour esquisser, devant les hommes du présent, leur devenir commun et manifester ce qui lie ensemble aujourd'hui. Les commémorations sont donc un marqueur de l'historicité, de la perception sociale du temps, tout autant qu'elles servent de fabrique identitaire » (Garcia, 2001, 33). Il y a dans cette consommation de passé tout autant une volonté de conjurer l'accélération des rythmes quotidiens et mondiaux que de collecter dans l'urgence les témoignages, les traces de modes de vie et modes de pensée en disparition, et de les remobiliser dans le présent. C'est donc bien l'identité comme construction durable et reproductible de soi qui est en jeu dans cette collecte d'objets hétéroclites qui ont comme seule nécessité que de référer au passé. Comme le relève M. Augé à propos de l'ouvrage de $\mathrm{P}$. Nora : "Le goût présent pour les commémorations traduit, par un paradoxe qui n'est que d'apparence, cette dissolution de la mémoire collective, en accusant le contraste entre un passé dont ne subsistent que des signes morts et un présent incertain de son identité » (M. Augé, rééd. 2001, p. 47). Remarque à laquelle répond le constat de F. Hartog: «le patrimoine en vient à définir moins que ce que l'on possède, ce que

\footnotetext{
${ }^{4}$ Cette réflexion s'appuie sur les travaux du groupe de recherche du laboratoire PACTE-Territoires, qui s'est saisi depuis deux ans de la question de la temporalité des territoires.
} 
l'on $a$, qu'il ne circonscrit ce que l'on est, sans l'avoir su, ou même sans avoir pu le savoir» (2003, p. 165).

Ces observations récurrentes se trouvent confrontées à une autre caractéristique du discours actuel sur le temps : la propension, parfois incertaine et hésitante, à se projeter dans le futur, mais dans un futur menaçant qu'il faudrait savoir anticiper. On pense ici tout autant à l'attente de l'opinion publique vis-à-vis de la prévention du risque ou du principe de précaution, que, dans un autre registre, aux politiques locales de prospective territoriale, ou encore aux mots d'ordre du développement durable à l'échelle mondiale. Ces attentes sont plus souvent, on le voit, à mettre en relation avec des représentations inquiètes du futur, qu'avec une projection optimiste dans un avenir meilleur.

Ces questionnements convergents ont amené la Géographie à repenser les temporalités, sous un angle différent du classique parallèle entre temps et espace. Il s'agit aujourd'hui d'interroger, à partir des territoires et de leurs dynamiques, l'imbrication entre temporalité et territorialité, à travers les nouvelles formes de mobilité, de proximité, d'appréhension de la distance. Cette réflexion rencontre tout particulièrement les analyses sur l'évolution des sociétés locales face à la mondialisation, la dilatation des réseaux, l'accélération des temps perçus. On peut alors voir dans les territoires des noyaux de stabilité ou d'inertie, ce qui a amené de nombreux géographes à annoncer la fin des territoires au nom de cette stabilité obsolète face à un monde de réseaux (ou au nom d'une conception du territoire essentiellement fondée sur un découpage national aujourd'hui partiellement dépassé). Cette position fait rapidement un sort à un concept encore d'actualité et utile à l'analyse, au nom d'une opposition quelque peu artificielle : les réseaux sont constitutifs des territoires, réseaux matériels ou idéels, hérités ou innovants, figés ou labiles. Il est vrai que les territoires ont longtemps été considérés comme des produits du temps long, inscrits dans une histoire, selon la formule de M. Marié : du «temps cristallisé ». Il faut sans doute aujourd'hui étudier d'autres territoires -sans oublier les premiers- ceux de l'urgence, du provisoire, voire de la précarité ; dans ce cas les processus de territorialisation sont eux mêmes caractérisés par l'éphémère de l'appropriation et de la consommation de signes. La "révolution des communications" et de la mobilité oblige à envisager des modes d'usage du territoire tout à fait nouveaux mais pas à renoncer à leur étude. Les formes de coprésence, les capacités d'ubiquité qu'apportent les technologies actuelles n'effacent pas -accentuent ?- le besoin d'ancrage; elles obligent l'observateur à adapter son regard et ses outils pour observer et chercher à comprendre des modalités complexes et mouvantes de rapport au territoire. Face à des temporalités fluctuantes, l'analyse porte moins sur des territoires, conçus comme des configurations figées, que sur la territorialité comprise comme une opération permanente d'ajustement et de réactualisation.

Le rapport au passé est dès lors lui aussi interrogé : le passé n'est pas ici un producteur "amont»d'identité et de territoire, mais un fonds actuel de ressources dans lequel les groupes puisent collectivement selon les modalités de la patrimonialisation et de la construction mémorielle. Il fournit des représentations du temps long et de l'ancrage symbolique, à des sociétés prises dans le temps court. La finalité est alors la construction d'une identité territoriale susceptible de se prémunir contre l'incertitude de l'avenir: "Tout se passe comme si l'homme avait besoin d'outils de navigation lui permettant de faire le point le plus souvent possible » (P-.A. Landel, 2002). Ces outils vont chercher plus volontiers leurs points de repère dans un passé gage de certitude que dans un présent insaisissable ou un futur menaçant. ${ }^{5}$

\footnotetext{
${ }^{5}$ Je renvoie ici à l'abondance des travaux sur les politiques patrimoniales des collectivités locales : projets de pays, villes moyennes, départements, réseaux et associations diverses autour des pays d'art et d'histoire, des
} 
On passe ainsi de sociétés -encore une fois sans les oublier- construites sur la transmission d'un héritage, d'une culture dite ancestrale, à des constructions territoriales fondées sur l'adhésion à une lecture du passé inventant ou bricolant (au sens positif de C. Levi-Strauss) des racines. Cette mémoire n'est pas figée, elle est ouverte à des ajouts, modifications, relectures, au fil de ces processus de réinterprétation.

Deux modalités sont distinguées ici, la patrimonialisation et la construction mémorielle. La patrimonialisation vise à sélectionner des objets dans le territoire, objets codifiés comme traces du passé (pas uniquement matériels: le monument, l'outil traditionnel, l'espèce floristique, mais objectivables : une technique, un savoir, une création artistique « vivante »), pour les ériger en bien commun à transmettre aux générations futures. Par ce biais, le collectif met en scène ses origines, son historicité, soit par la muséification (écomusée, maison du patrimoine...), soit par une préservation in-situ, soit par une valorisation économique et sociale qui maintient l'objet dans ses fonctionnalités, ou dans des fonctionnalités redéfinies. Relevons ici le terme "tradition", qui fleurit dans les discours sur le patrimoine: la valorisation du traditionnel s'appuie sur une représentation d'un passé indéterminé, mais collectif et consensuel, et répondant aux attentes du présent ${ }^{6}$; selon la formule provocatrice de B. Latour, « On ne naît pas traditionnel, on le devient en innovant beaucoup » (1997, p.103).

La tradition est très souvent accompagnée d'une autre expression clé de la valorisation du passé, 1'"authenticité", qui partage dans l'usage courant, le même caractère consensuel : est authentique ce qui est décrété comme tel, c'est-à-dire ce qui répond à la représentation d'un passé positif et le confirme. L'authenticité possède en outre la fonction supplémentaire de modalité d'évaluation et de légitimation de ce passé, et du coup du patrimoine.

La construction mémorielle est elle mobilisée pour donner du sens et créer un lien collectif, une cohérence entre ces objets et entre ces objets et une configuration du monde. Elle fournit un cadre cognitif et symbolique qui inscrit un collectif dans la durée, au nom du partage d'une même lecture du passé déroulé dans un même territoire, et elle conforte ainsi son identification. Dans la profusion des recours à la mémoire, le géographe ne peut qu'être attentif à la fragmentation actuelle en une multitude de constructions locales, mettant en scène des personnes ordinaires, des objets banals dans des lieux quotidiens. Depuis le début des années quatre-vingts, les collectivités territoriales se sont emparées de ces outils de légitimation et en ont diversifié à l'infini les modes et les lieux. En cédant au goût de la formule, on peut dire que la construction mémorielle combine la mise en récit d'un passé fédérateur, sa mise en scène dans un territoire où inscrire des traces, et une mise en perspective avec un territoire souhaitable. P. Garcia, toujours à propos des commémorations, insiste également sur cette articulation: «L'engagement des collectivités territoriales repose sur la conviction unanime que la capacité à relever des défis du futur est proportionnelle à l'ancrage dans l'histoire, à l'existence de traditions particulières qu'il faut donc redécouvrir et, au besoin, inventer." » (Garcia, 2001, 36).

écomusées, etc. Voir le $\mathrm{n}^{\circ}$ spécial de Montagnes méditerranéennes: "Patrimoines, Territoires et Création d'activités", $2002, \mathrm{n}^{\circ} 15$.

${ }^{6}$ J'évoquerai à titre d'exemple, dans le registre architectural, une pratique qui illustre bien cette idée de fonds de ressources patrimoniales : la constitution des «styles locaux» en architecture. Leur élaboration ne s'appuie pas sur une exploration des matériaux et techniques anciens mais sur la sélection «à la carte » dans l'ensemble des ressources possibles d'éléments qui, combinés, formeront un style ; celui-ci est souvent fait d'emprunts à des techniques, des matériaux et des origines hétéroclites, mais qui rencontrent les goûts architecturaux du moment. C'est ainsi que les lauzes sont devenues depuis une trentaine d'années le revêtement par excellence des toitures des Alpes du nord, particulièrement bien représenté dans les secteurs qui ont les moyens d'imposer une norme coûteuse, les stations de ski. Les débats locaux sur ces types et leurs critères apparaissent de plus en plus fréquemment comme des occasions de controverses vives et de cristallisations des oppositions. 
Ceci amène à envisager l'identité territoriale sous un angle dynamique, comme une construction qui ne puise pas exclusivement dans la lecture du passé, contrairement à une tendance souvent " passéisante » des analyses de l'identité, mais qui vise la durée. Elle joue ainsi sur les deux versants : celui de l'ancrage et celui de la projection, et fait le pari de leur cohérence. C'est pourquoi il est généralement plus pertinent de réfléchir en termes de processus d'identification à un territoire qu'en termes d'identité territoriale, celle-ci laissant penser qu'il est envisageable de la saisir pour la soumettre à l'observation et à l'analyse.

L'identification peut ainsi se construire sur des arguments mettant en avant la modernité, ou l'innovation, la durabilité, ou même la capacité à affronter le futur. L'exemple le plus frappant en sont les revendications de nombreuses sociétés locales, qualifiées de traditionnelles pour ne pas dire primitives, et qui affirment aujourd'hui leur capacité d'adaptation au monde actuel, leur conformité notamment aux exigences de durabilité, et du coup leur modernité retrouvée.

Le discours sur le territoire combine ainsi des lieux assignés au passé et des lieux porteurs d'innovations et cherche à en démontrer l'interdépendance.

\section{Mémoire des lieux et effet géographique}

Le renversement de l'expression nous conduit à la question de l'inscription des lectures du passé dans le territoire et cherche à mettre en lumière l'intérêt de l'approche géographique du terme lieu, au sens que lui donne A. Berque (2003).

Si les principaux « théoriciens » de la mémoire, notamment $\mathrm{M}$. Halbwachs ou $\mathrm{P}$. Ricoeur, ont relativement peu développé la question de l'inscription spatiale de la mémoire, celle-ci est néanmoins présente dans les deux œuvres. Halbwachs pour affirmer que la mémoire ne peut être que collective, donc située dans un milieu collectivement partagé ; Ricoeur pour insister sur la notion de " place ": « De façon générale, tout groupe assigne des places. C'est de cellesci que l'on garde ou forme mémoire (...). (Les souvenirs) nous font voyager de groupe en groupe, de cadre en cadre, tant spatiaux que temporels. Reconnaître un ami sur un portrait, c'est se replacer dans les milieux où nous l'avons vu » (P. Ricoeur, 2000, 148-149). Ou tout simplement pour rappeler le sens de la formule «J'y étais » : qui appelle au témoignage mais affirme aussi que l'événement raconté est situé dans un lieu. (P. Ricoeur, 2000).

Néanmoins, il s'agit là d'insister sur la localisation du souvenir, le lieu dont on se souvient, plutôt que sur le lieu où l'on se souvient ; les deux n'étant pas forcément confondus. Les lieux dont on se souvient posent le décor de la mise en récit; non seulement ils ne nécessitent pas la présence sur place mais au contraire ils cherchent à figer l'image telle qu'elle subsiste dans les souvenirs. Les lieux où l'on se souvient ré-ancrent, eux, la mémoire dans le territoire actuel; on s'y déplace, on vient voir. Ce sont les seconds qui nous intéressent ici.

Dans la vie publique, les pratiques mémorielles ont donné une ampleur toute particulière aux « lieux de mémoire », lieux où l'on se souvient: monument, stèle, mémorial, parcours de mémoire... Ceux-ci tendent à devenir avec le temps des lieux d'histoire, quand les témoins disparaissent, et que le récit se perd au profit d'une explication distancée. C'est là un des enjeux du mémorial: comment faire en sorte que la mise en scène fasse perdurer la mémoire, sa capacité à émouvoir et à mobiliser, et quel est alors le rôle du lieu et de ses dimensions sensibles?

On observe des processus de cristallisation de la mémoire dans certains lieux: lieux de rassemblements, de cérémonies, de rites, nommés et pratiqués comme tels. C'est bien là une fonction essentielle du lieu en géographie, celle qui fait converger en un point de taille variable, des processus symboliques qui prennent une forme propre, une matérialité. 
Je citerai un exemple, frappant parce que non organisé, de cette volonté de signaler le lieu: la pratique qui semble se diffuser de marquer d'un bouquet de fleurs, soigneusement renouvelé, le long de la route, ou d'une stèle funéraire en montagne, les lieux d'accident (E. Petit, 2004); il s'agit là d'une mémoire familiale le plus souvent, mais qui recherche une forme de reconnaissance collective, notamment dans le cas des stèles. Ces pratiques incitent à valider l'idée que la mémoire a besoin de lieux pour subsister, pour être partagée ; pas simplement des lieux éphémères de rassemblement mais des lieux identifiés comme tels et qui permettent d'inscrire dans le territoire cette mémoire collective. L'inscription doit donc permettre de lutter contre l'oubli, et aussi dans les cas qui précèdent contre l'ignorance des autres: ignorance intolérable de l'événement qui s'est déroulé là.

C'est à travers la fréquentation de ces lieux, conjuguant symbolique et matérialité de l'inscription, que le groupe affirme son existence en tant que groupe, et y puise des fondements à son identification.

« Le souvenir cristallisé dans un objet localisé ou dans un lieu constitue en effet l'évidence de l'existence et de la cohésion groupales. Leur fréquentation quotidienne ou du moins régulière renforce leur efficacité car elle est l'affirmation récurrente de la lecture commune du temps par le groupe. Cependant, il n'est pas nécessaire que le lieu dépositaire de la mémoire du groupe, et par la suite de son existence, soit physiquement présent ; il suffit que son existence soit connue et attestée » (Mauz, 1997, 23).

Ces lieux de mémoire ne sont pas choisis par le simple fait que " ça s'est passé là ", l'intérêt est précisément de comprendre pourquoi certains sont sélectionnés, codifiés, valorisés, et deviennent parfois des "hauts-lieux". " Ces points du monde où l'on peut penser qu'on pourrait, du fait de traditions religieuses ou de la qualité d'un site, ou d'un ciel, atteindre mieux qu'ailleurs au rapport à soi qu'on recherche »(Bonnefoy, 1990). Le lieu, par sa matérialité naturelle et/ou construite offre des conditions favorables à cette cristallisation : configuration particulière du site, particularité du relief ou de la végétation, forme de bâti originale... Devenus lieux de mémoire collectifs, ils ne sont pas pour autant figés dans une signification et une fonction rituelle définitive : ils sont toujours susceptibles d'être remaniés ou oubliés. Néanmoins, ils subissent là une forme d'objectivation, ils sont localisés, nommés, et intégrés dans une catégorie bien spécifique d'objets spatiaux, qui implique des pratiques elles aussi spécifiques. F. Walter, à propos des figures paysagères de l'identité nationale relève que: " En effet, il existe des paysages qui condensent en un espace restreint tout ce qu'on attribue à l'identité nationale. Ce sont de véritables territoire-musées où se mêlent nature et histoire » (2003, p. 340). Instrumentalisés par les autorités comme tels, ces paysages sont protégés, soignés, réhabilités selon cette logique mémorielle et identitaire: « Il y aurait donc une sorte de "grammaire" du paysage qu'on s'efforcerait d'appliquer pour rendre immédiatement lisible l'adéquation avec les valeurs nationales » (id. p. 339). Et il cite comme exemple la "Prairie de Rütli" au bord du Lac des Quatre Cantons en Suisse, érigée en berceau de la Confédération helvétique.

Le lieu de mémoire cristallise ${ }^{7}$, on l'a dit, une mémoire; mais celle-ci peut renvoyer de manière diffuse à l'ensemble du territoire, par un processus de type métonymique ${ }^{8}$.

Deux exemples plus développés permettent d'expliciter plusieurs de ces aspects croisés de la mémoire des lieux. Il s'agit pour le premier du Hameau de Valchevrière dans le massif du Vercors. Ce lieu est aujourd'hui signalé, visité et entretenu comme l'un des hauts-lieux de la Résistance dans le Vercors ; il est l'aboutissement de la route de Valchevrière, petite route de montagne, étroite et escarpée, transformée en chemin de croix dès la fin des années 1940.

\footnotetext{
${ }^{7}$ On voit que $\mathrm{F}$. Walter préfère la condensation; succès des métaphores atmosphériques.

${ }^{8} \mathrm{Ou}$, pour être plus précis, par effet de synecdoque.
} 
Cette route marque un des combats les plus meurtriers entre la Résistance et les troupes allemandes lors de l'attaque du maquis pendant l'été 1944, les maquisards reculant et perdant progressivement leurs positions. En contrebas de cette route se trouve le hameau, non pas lieu de combats mais qui fut entièrement détruit par les Allemands en représailles. Les ruines ont été conservées en l'état, quelques ouvrages évitant l'effondrement des murs, des fauches et abattages réguliers freinant la progression de la forêt dans la clairière.

Avant la guerre, ce hameau perché sur un versant escarpé était pratiquement abandonné car éloigné des nouvelles routes du Vercors, difficile d'accès; l'habitat se réduisait à quelques bergeries et habitats temporaires d'estive. C'est le souvenir des combats de la Résistance qui l'a extrait, bien après la fin de la guerre, d'une histoire révolue pour l'ériger en " village martyre » et il est aujourd'hui connu et visité à ce titre. Il semble que la mémoire de telle qu'elle est transmise aujourd'hui s'appuie autant sur le récit de la prise du massif, racontée sur place par des panneaux, que sur la perception du lieu : le site surplombant les gorges, la configuration en clairière dans laquelle se dispersent les ruines, les marges forestières contribuent tout autant à faire de Valchevrière un haut-lieu. Mise en récit, mise en scène, c'est bien par l'effet de lieu qu'il s'agit aujourd'hui de conjurer l'oubli : les générations passant, les souvenirs de la Résistances se perdent au profit d'une histoire qui est racontée in situ et dont la force est amplifiée par le décor. Cet exemple illustre les interactions entre paysage et mémoire : le paysage est perçu à travers le filtre de cette fonction de haut-lieu, et en retour, il contribue à cette codification du lieu.

Un autre lieu, proche dans l'espace mais dans un registre différent, permettra d'illustrer un autre lien entre mémoire et territoire. Le lieu-dit "Champs de la Bataille » situé également dans le Vercors, tient son nom d'un épisode ancien et oublié de l'histoire locale : au début du $\mathrm{XV}^{\circ} \mathrm{s}$., une bataille opposa l'évêque de Die et le Comte de Sassenage, seigneurs locaux, autour de la possession de forêts et d'alpages. Le toponyme conservé sur les cartes et dans les usages, pour désigner une clairière, était en voie de perdre toute signification, voire d'être confondu avec un lieu de combat de la Résistance. Le développement touristique, le tracé des sentiers de Grande Randonnée et l'effort de signalétique qui suivit, amena les autorités à installer des panneaux explicatifs le long des itinéraires, faisant largement référence aux deux ressources principales du patrimoine local: la nature et l'histoire. Les habitants retrouvèrent ainsi la signification du toponyme et aucun aujourd'hui ne manque de fournir l'explication, souvent complétée de son propre chef, au visiteur. On relève ici le rôle moteur joué par les toponymes dans l'appropriation d'un territoire pas ses usagers, ici tant l'habitant que le visiteur curieux. Ce lieu et ce nom permettent de découvrir une histoire lointaine et oubliée et de l'intégrer dans une mémoire actuelle tout à fait vivante, parce qu'elle nourrit la connaissance du territoire par ses habitants. Elle s'appuie en outre sur deux des composantes principales, l'une regardant vers le passé et l'autre vers le futur, de l'identification au territoire: la forêt, ressource économique traditionnelle et facteur d'appropriation, et le tourisme orientation actuelle des activités locales. On observe dans ce second cas une application inverse du processus métonymique évoqué plus haut: à partir d'un toponyme et d'un lieu, la mémoire se diffuse sur l'ensemble du territoire.

La construction mémorielle est donc indissociable du discours sur le territoire, et elle organise sa lecture des lieux dans une configuration à la fois symbolique et pratique. Construction mémorielle et patrimonialisation interagissent ici étroitement. Quand un groupe retrouve et identifie des traces dans le territoire, qu'il interprète en référence au passé, ces traces sont versées au patrimoine collectif et devront être transmises comme telles. Jusque là parfois ténues et anonymes, elles deviennent des signes, que l'on cherche à rendre explicites, univoques et qui sont dès lors intégrés dans la gestion globale du territoire: le hameau de 
Valchevrière doit être entretenu en tant que lieu de mémoire; pas question de laisser le Champ de la Bataille envahi par les friches ou noyé dans un nouvel équipement de loisirs. On voit ailleurs des collectivités territoriales, en mal de patrimoine dans des territoires "sans histoire", inventer des signes d'un passé trop peu mobilisable et les mettre en scène ${ }^{9}$.

En effet, si l'on envisage la question maintenant du point de vue de l'action collective et des politiques publiques, elles aussi attentives aux revendications mémorielles et produisant ellesmêmes de la mémoire collective, se pose la question de la finalité de ces productions. Il s'agit bien ici d'identifier une trajectoire des territoires entre mobilisation du passé et projet : la mémoire est chargée de faire le lien, de définir une cohérence, et, pourquoi pas, d'ouvrir des perspectives, le patrimoine est chargé de les rendre lisible.

Ce questionnement peut être utilement éclairé par la notion d'effet géographique telle qu'elle a été proposée par B. Debarbieux: «le processus par lequel l'identification d'un objet spatial et son inscription dans une catégorie d'objets similaires rend possible et efficace le déploiement d'un ensemble d'actions concrètes sur la matérialité de cet objet ou de certains de ses éléments » (2004, p. 27). Dans le cas présent, les processus de construction mémorielle amènent à isoler et catégoriser des objets spatiaux chargés de valeurs patrimoniales et identitaires : monuments, lieux de mémoire, itinéraires, techniques, matériaux... Ce statut infléchit dès lors les logiques de gestion et d'intervention: conservation, préservation, maîtrise des évolutions, valorisation, requalification ...

Si l'on poursuit plus avant la problématique de l'effet géographique, la question se pose de la pérennisation de ces actions et de l'évolution conjointe des objets ainsi catégorisés et des logiques d'intervention. "En retour, un ensemble de transformations totalement indépendantes de la volonté humaine et parfois imperceptibles font évoluer le référent de l'objet, dans un sens qui peut-être attendu ou redouté. L'effet géographique s'inscrit donc dans une mise en tension de ces intentions, de ces actions et de ces processus qui, tous ensemble, configurent et dénaturent à la fois les objets dont est fait l'espace géographique. » (id., p. 29). De la même manière que la mémoire est sans cesse revisitée, réactualisée, bricolée, les configurations territoriales elles aussi évoluent et dès lors réorientent les logiques d'action; et les objets eux-mêmes «vivent leur vie d'objet», selon les processus physiologiques : envahissement des lieux de mémoire par la forêt, écroulement des murs... pour reprendre les exemples qui précèdent. Une trace, un moment érigée en signe patrimonial doit être entretenue dans sa signification, au risque de redevenir trace, voire de s'effacer aux yeux des usagers.

Si la mémoire cherche à construire de la continuité entre passé et projection dans le futur, si elle permet de garantir la cohérence de la construction identitaire au fil de cette trajectoire, elle implique néanmoins que cette construction aboutisse à un consensus, pas tant sur le contenu de cette mémoire que sur les usages que l'on en fait, sur le projet que cette construction sous-tend. C'est à cette condition que l'on peut dire que la mémoire est tournée vers l'avenir, est une mémoire ouverte et non le signe d'un repli.

Le Vercors offre à nouveau une illustration de constructions divergentes autour de la mémoire de la Résistance. Les hauts-lieux du Maquis se trouvent en effet érigés au rang de mémoire nationale et des «injonctions de mémoire » viennent régulièrement orienter la politique locale : rénovation de sites, actions pédagogiques, construction d'un Mémorial. Si l'on se situe à l'échelle des populations locales, la rareté des références à cette histoire est un constat

\footnotetext{
${ }^{9}$ Les communes de banlieue ou les villes moyennes offrent souvent des exemples de politiques patrimoniales travaillant sur des artefacts de ce type: mobilier urbain "traditionnel", réfection des façades avec crépis "à l'ancienne" etc. Voir dans ce même ouvrage la contribution de S. Périgois.
} 
fréquemment renouvelé : la volonté d'oubli au quotidien de ces moments douloureux de la mémoire familiale prédomine, et il est clair qu'elle n'est pas constitutive d'une identité locale (A. Sgard, 1997). A l'échelon intermédiaire des collectivités territoriales, du parc régional et des associations, la gestion de ces lieux de mémoire, des itinéraires, des équipements se pose en termes très concrets de coût et de responsabilité. Mais on identifie aussi de façon moins explicite des enjeux symboliques à travers les lectures diverses que cette mémoire mobilise: pour les uns c'est une mémoire douloureuse, qu'il faut transmettre dans les figures dramatiques du martyre et du sacrifice ; pour d'autres ce doit être aussi la mémoire glorieuse des combattants, mise en scène sur d'autres registres: celui du courage, de l'héroïsme (Vergnon, 2002). Ces choix amènent à valoriser des moments, des lieux, des acteurs différents, dans des mises en récits et des mises en scènes différentes.

Cet exemple montre la difficulté à construire un projet autour d'une mémoire pourtant très présente, a priori riche et reconnue, quand échelles et lectures s'emboîtent et interfèrent ; il illustre la difficulté que rencontrent les collectivités territoriales pour bâtir une politique commune autour du patrimoine et de la mémoire, car au-delà, se pose pour les gestionnaires de ces lieux la question de la finalité : se souvenir, mais pour qui et pour quoi ? ${ }^{10}$

On voit donc que l'enjeu n'est pas le contenu de la mémoire, ni les objets de la patrimonialisation, mais bien le projet qu'ils sont censés porter et exprimer. $\mathrm{Si}$, comme nous le disions plus haut, les politiques patrimoniales et les discours publics mobilisant la mémoire visent à conforter une identité malmenée, l'inscription de cette mémoire dans le territoire, et plus particulièrement dans des lieux, cherche à afficher et à faire partager par l'ensemble du groupe cette lecture du passé commun, voire à imposer une seule lecture. Les conflits graves ou anodins autour du marquage du territoire confirment bien que l'inscription n'est pas conçue pour être négociée et reformulée mais pour durer. La mobilisation de la mémoire devient un argument clé des discours sur le futur, dont on relève souvent le caractère incantatoire: ils dessinent un avenir des territoires pour mieux légitimer les acteurs qui le produisent et affirmer leur longévité. Il est sans doute plus facile d'inscrire dans les lieux ce que nous sommes au nom de ce que nous avons été, que d'imaginer ce que nous serons et se risquer à une projection dans le futur.

\section{Conclusion}

La thématique du patrimoine et de la mémoire envahit, on l'a dit, les discours politiques et aménagistes, notamment dans le cadre des pays, des inter-communalités, des politiques urbaines, mais aussi à l'échelle internationale, dans les discours sur le développement et la participation. Au-delà de l'effet de mode, les contextes, les modalités de cette mobilisation de la mémoire et les argumentaires dans lesquels elle s'insère demandent à être analysés, pour fournir des éléments pertinents d'analyse des territorialités et de leurs dynamiques, ainsi que des logiques d'acteurs. Ne serait-ce que pour y démêler les modèles de références, les cadres idéologiques et les valeurs véhiculées.

La consommation de passé par les politiques territoriales a été abondamment mise en lumière; il est frappant de constater combien les thématiques les plus explicitement tournées vers le futur, comme les réflexions de prospective territoriale ou d'élaboration des « best practices »

\footnotetext{
${ }^{10}$ Entretenir des lieux, préserver des objets, mais pour transmettre quel message ? Un des lieux récemment créés à Vassieux-en-Vercors, le « Jardin de la mémoire », propose une réponse : se souvenir pour demain ; du site, un ancien cimetière, le visiteur regarde, à travers des stèles translucides, le plateau et les crêtes du Vercors, le village et les routes, les habitants d'aujourd'hui. A travers les diverses générations de lieux de mémoire créés depuis le lendemain de la guerre dans le Vercors, on voit ainsi les objets et les logiques d'action se succéder.
} 
pour le développement durable, font appel à la question de la mémoire locale. Dans le premier cas, alors que la «théorie » de la prospective insiste sur la rupture vis-à-vis du présent et du passé au nom de la nécessaire projection dans des futurs potentiels, sa mise en œuvre se manifeste sur le terrain par des références récurrentes aux mémoires locales : tantôt les mémoires politiques, les mémoires de l'action, porteuses d'enseignement et d'une culture locale de l'aménagement, soit plus classiquement, les mémoires collectives nécessaires à l'ancrage territorial. Dans le cas du développement durable, la prise en compte nécessaire des populations locales qu'il préconise se traduit de plus en plus souvent pas la (re)découverte de ce que l'on appelle tantôt savoirs locaux, tantôt savoirs vernaculaires, en tout cas connaissance endogène du territoire ; la question qui se pose alors est d'identifier l'ancrage de ces connaissances, de ces techniques, dans un cadre cognitif et symbolique, autrement dit une mémoire vivante permettant le maintien, l'actualisation et l'opérationnalisation de ces savoirs locaux.

Au constat mi-amer de P. Nora et de ses co-auteurs relevant la disparition de bon nombre de ces lieux dont ils faisaient l'inventaire : " On ne parle tant de mémoire que parce qu'il n'y en a plus », répondons par le déplacement du regard et de l'échelle : la mémoire s'éparpille, se diversifie, et par le changement de perspective: on ne parle tant de mémoire que parce les images du futur se brouillent.

\section{Bibliographie}

AUGE M., Pour une anthropologie des mondes contemporains, Flammarion, col. Champs, 1997, rééd. 2001,196 p.

BERQUE A., article «Lieu », LEVY J. \& LUSSAULT M. (dir.), Dictionnaire de Géographie et de l'espace des sociétés, Belin, 2003, p. 555-556 et bibliographie.

CHESNAUX J., Habiter le temps. Présent, passé, futur : esquisse d'un dialogue politique, Bayard éd., 1996.

DEBARBIEUX B., FOURNY M.-C., L'effet géographique. Construction sociale, appréhension cognitive et configuration matérielle des objets géographiques, Publ. MSHALPES, 2004, 248 p.

GARCIA P., "Exercices de mémoire? Les pratiques commémoratives dans la France contemporaine ", in "La mémoire, entre histoire et politique", Cahiers français, $\mathrm{n}^{\circ} 303$, Juillet-Août 2001, 33-39.

GARCIA P., article « Mémoire sociale », LEVY J. \& LUSSAULT M. (dir.), Dictionnaire de Géographie et de l'espace des sociétés, Belin, 2003, p. 602-604.

GRANET-ABISSET A.M., "Mémoire alpine et construction européenne ", Revue de Géographie alpine-Journal of alpine research, n², 2004, p. 39-48.

HALBWACHS M., La mémoire collective, A. Michel, 1950-rééd. 1997, 298 p.

HARTOG F., Régimes d'historicité, présentisme et expérience du temps, Ed. Le Seuil, col. Librairie du $\mathrm{XXI}^{\circ} \mathrm{s}$., 2003, 256p. 
LANDEL P.-A., « Patrimoines, territoires et création d'activités : vers une mise en réseau des acteurs », Montagnes méditerranéennes, $\mathrm{n}^{\circ} 15,2002$, p. 9-13.

LAÏDI Z., Le temps mondial, le sacre du présent, 2003.

LATOUR B., Nous n'avons jamais été modernes. Essai d'anthropologie symétrique, Ed., La Découverte, col. Sciences humaines et sociales, 1997, $210 \mathrm{p}$.

NORA P. dir., Les lieux de mémoire, Gallimard, Bibliothèque des sciences humaines, 1984-1993.

MAUZ I., Bouquetin, Chamois et Territoires : Recherches sur le rôle du Bouquetin et du Chamois dans la configuration symbolique de l'espace en Vanoise, Grenoble, Cemagref Université Joseph Fourier, Mémoire de DEA, 1997.

PETIT E., «Figures de la haute montagne au service de la mémoire collective à Chamonix », DEBARBIEUX B., FOURNY M.-C. (dir.), L'effet géographique. Construction sociale, appréhension cognitive et configuration matérielle des objets géographiques, Publ. MSH-ALPES, 2004, p. 89-114.

PIVETEAU J.-L., « Le temps est-il un lieu de mémoire ? », L'espace géographique, n², 1995, p. 113-123.

RICOEUR P., La mémoire, l'histoire, l'oubli, Ed. Le Seuil, col. Point-Essais, 2000, 680 p.

RAUTENBERG M., «La patrimonialisation, entre appropriation sociale et désignation institutionnelle », DEBARBIEUX B., FOURNY M.-C. (dir.), L'effet géographique. Construction sociale, appréhension cognitive et configuration matérielle des objets géographiques, Publ. MSH-ALPES, 2004, p. 71-88.

SGARD A., Paysages du Vercors, entre mémoire et identité, Revue de Géographie alpine, col. Ascendances, 1997, $168 \mathrm{p}$.

VERGNON G., Le Vercors, histoire et mémoire d'un maquis, Ed. de l'atelier, Paris, 2002, 